\title{
Identification of Crack Location in Beam Structures Using Wavelet Transform and Fractal Dimension
}

\author{
Yong-Ying Jiang, ${ }^{1,2,3}$ Bing Li, ${ }^{1,2}$ Zhou-Suo Zhang, ${ }^{1,2}$ and Xue-Feng Chen ${ }^{1,2}$ \\ ${ }^{1}$ The State Key Laboratory for Manufacturing Systems Engineering, Xi'an 710049, China \\ ${ }^{2}$ School of Mechanical Engineering, Xian Jiaotong University, Xian 710049, China \\ ${ }^{3}$ School of Mechanical and Electrical Engineering, Wenzhou University, Wenzhou 325035, China \\ Correspondence should be addressed to Bing Li; bli@mail.xjtu.edu.cn
}

Received 13 December 2014; Accepted 20 January 2015

Academic Editor: Yanxue Wang

Copyright (C) 2015 Yong-Ying Jiang et al. This is an open access article distributed under the Creative Commons Attribution License, which permits unrestricted use, distribution, and reproduction in any medium, provided the original work is properly cited.

\begin{abstract}
Identification of structural crack location has become an intensely investigated subject due to its practical importance. In this paper, a hybrid method is presented to detect crack locations using wavelet transform and fractal dimension (FD) for beam structures. Wavelet transform is employed to decompose the mode shape of the cracked beam. In many cases, small crack location cannot be identified from approximation signal and detailed signals. And FD estimation method is applied to calculate FD parameters of detailed signals. The crack locations will be detected accurately by FD singularity of the detailed signals. The effectiveness of the proposed method is validated by numerical simulations and experimental investigations for a cantilever beam. The results indicate that the proposed method is feasible and can been extended to more complex structures.
\end{abstract}

\section{Introduction}

Crack identification has gained increasing attentions from the scientific and engineering domains since the unpredicted structural failure may cause catastrophic, economic, and life loss. In the past several decades, many crack detection methods and techniques have been developed. Among them, nondestructive detecting technique is reliable and effective in maintaining safety and integrity of structures $[1,2]$. However, most nondestructive crack identification methods, such as ultrasonic methods and X-ray methods, are costly and timeconsuming for large structures. In the last several decades, a lot of research efforts have been made to develop an effective approach to detect cracks in structures. The vibration-based crack detection methods are developed to overcome these difficulties $[3,4]$. The fundamental idea of vibration-based crack identification is to detect the crack-induced changes in the physical properties, which will lead to detectable changes in mode properties [5-7].

Natural frequency-based crack identification methods employ the natural frequency change as the basic feature [8-11]. The natural frequency-based method is attractive because the natural frequencies can be conveniently measured from just a few accessible points. By aid of the frequency, many crack identification methods were developed [12-16]. Although frequency is usually regarded as an easyobtained characteristic with satisfying accuracy even for complex structures, its properties of lumped parameter greatly restrict wide application of frequency-based crack detection methods. Another limitation is that the crack identification problem is often ill-posed even without noise pollution, which leads to nonuniqueness of the solutions of crack location and severity. First, it is obvious that crack with same severity in symmetric locations of a symmetric structure will cause identical frequency changes. Furthermore, crack with different severity in different locations can also produce identical changes in a few measured natural frequencies.

Crack detection methods have been developed based on measured mode shapes directly or indirectly [17-19]. These methods are roughly divided into two categories. The traditional methods based on mode shape try to construct a relationship between crack location and mode shape change by a finite element method or experimental test. However, 


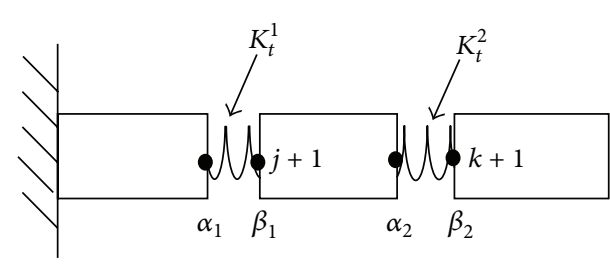

(a)

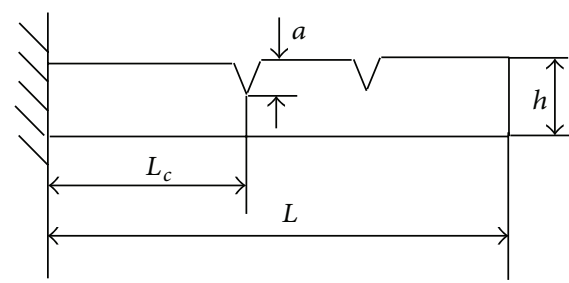

(b)

FiguRE 1: Cracked cantilever beam: (a) geometry, (b) model of cracked beam.

they need mode shape data from intact structures. Because of the development of modern signal processing techniques, modern signal processing methods can be employed to extract singularity information of the mode shape in the cracked structures $[16,20-22]$. Singularity detection using mode shape is attractive mainly because it is possible to detect crack location without the a priori knowledge in the cracked zones. However, small crack has a minor effect on the mode shape. So singularity information of the mode shape is not identified directly without further analysis. Wavelet transform is a useful tool to detect local singularity characteristic of many different kinds of signals through decomposing the signals [23-27]. These methods treat mode shape data as a signal in spatial domain, and they use spatial wavelet transform technique to detect the irregularity of the signal caused by crack. To detect the singularities using wavelet transform, the mode shapes of cracked structure should be acquired first. Then the wavelet coefficients are calculated from the mode shapes, and the wavelet coefficients are plotted in the full region for each level of wavelets. Finally the distributions of the wavelet coefficients at each level are examined, and the peaks or sudden changes in the distributions of the wavelet coefficients are applied to locate the crack positions. The limitation of wavelet transform methods is very common; that is, there are serious boundary effects. In many cases, the irregularity of the signal caused by crack is not detected using wavelet transform alone.

As an efficient index of crack, fractal dimension is also introduced to detect crack for beam and plate structures by Hadjileontiadis et al. $[28,29]$. Due to its simplicity of the fractal dimension (FD) evaluation and its robustness against noise, a FD-based technique has been developed to identify cracks of structures [28-31]. Crack location is determined by a peak on the FD curve through the local irregularity of the fundamental mode shape introduced by the crack. If the higher mode shapes were considered, this method might give misleading information.

In the present work, a hybrid method is presented to detect crack locations based on wavelet transform and fractal dimension (FD) for beam structures, which can enhance the high sensitivity to the singularities induced by structural crack. Using Db4 wavelet decomposition, approximation signal and wavelet detailed signals of the mode shape are obtained. Then FD estimation method is used to calculate FD parameter of detailed signal. The crack location can be determined by the abrupt change of the FD along the beam length. Numerical simulation and experimental investigation are performed to testify the present method. In the numerical simulation, the mode shapes of the beam with crack are obtained based on wavelet-based Euler beam elements using $\mathrm{B}$-spline wavelet on the interval (BSWI), whereas in the experimental investigation, the mode shapes are measured using Polytec Vibrometer PSV-400.

\section{BSWI Finite Element Model of Cracked Beam}

Figure 1(a) shows a model of cracked cantilever beam with dimensions of length $L$ and uniform cross section $b \times h$ (where $b$ is the width and $h$ is the depth of beam), two open cracks of depth $a_{i}$ locates at $L_{c i}$ away from the clamped end, where the subscript $i$ expresses the serial number of cracks. Since the linear rotational spring model can describe open crack effectively, present work is based on this model (as show in Figure 1(b)). The stiffness of rotational springs $K_{t}$ can be written as

$$
K_{t}^{i}=\frac{b h^{2} E}{72 \pi(a / h) f(a / h)},
$$

where $E$ is the modulus of elasticity of beams, $h$ is the depth of beams, and $f(a / h)$ is a dimensionless local compliance function expressed as follows [32]:

$$
\begin{aligned}
f\left(\frac{a}{h}\right)= & 0.6384-1.035 \frac{a}{h}+3.7201\left(\frac{a}{h}\right)^{2}-5.1773\left(\frac{a}{h}\right)^{3} \\
& +7.553\left(\frac{a}{h}\right)^{4}-7.332\left(\frac{a}{h}\right)^{5}+2.4909\left(\frac{a}{h}\right)^{6} .
\end{aligned}
$$

To express briefly, we denote two dimensionless parameters to describe cracks: relative crack size $\alpha_{i}=a_{i} / h$ and normalized location $\beta_{i}=L_{c i} / L$.

As shown in Figure 1(b), the continuity conditions at crack positions 1 and 2 indicate that the left nodes $j, k$ and the right nodes $j+1, k+1$ have the same transverse displacement, namely, $u_{j}=u_{j+1}$ and $u_{k}=u_{k+1}$, whereas the rotations $\theta_{j}$, $\theta_{k}$ and $\theta_{j+1}, \theta_{k+1}$ are connected through the cracked stiffness submatrix $\mathbf{K}_{s}^{i}(i=1,2)$ as follows:

$$
\mathbf{K}_{s}^{i}=\left|\begin{array}{cc}
K_{t}^{i} & -K_{t}^{i} \\
-K_{t}^{i} & K_{t}^{i}
\end{array}\right| .
$$


The global stiffness matrix $\mathbf{K}$ and mass matrix $\mathbf{M}$ of BSWI Euler beam can be obtained according to the literature [33], in which the specific formulas about wavelet-based modeling for beam using BSWI bases are shown. According to relative locations $\beta_{1}$ and $\beta_{2}$ of the crack, we can assemble cracked stuffiness submatrix $\mathbf{K}_{s}^{i}(i=1,2)$ into the global stuffiness matrix $\mathbf{K}$ in the corresponding place. The global mass matrix $\mathbf{M}$ of the cracked beam is the same as the uncracked one.

The free vibration problem of plate is expressed as a generalized eigen-problem:

$$
\left(\mathbf{K}-\omega^{2} \mathbf{M}\right) \mathbf{X}=0
$$

where $\omega$ is the natural frequency and $\mathbf{X}$ is the mode shape. Submitting matrix $\mathbf{K}$ and matrix $\mathbf{M}$ into (4), the natural frequency and mode shape of beam vibration are obtained.

In the present, the command EIG in software MATLAB is employed to solve (4). The QR-algorithm and QZ-algorithm are automatically selected to solve the symmetry and nonsymmetry generalized eigenvalue problems [34].

\section{Fractal Dimension}

The concept of FD and its relevant mathematical model were originally introduced by Mandelbrot [35]. The proposed approach adopts Katz's estimation of the FD. According to Katz [36], the FD of a curve defined by a sequence of points is estimated by

$$
\mathrm{FD}=\frac{\log _{10}(n)}{\log _{10}(d / L)+\log _{10}(n)},
$$

where $n$ is the number of steps in the curve, $d$ is the diameter estimated as the distance between the first point of the sequence $P_{1}$ and the $i$ th point $P_{i}$ of the sequence that provides the farthest distance; and $L$ is the total length of the curve or the sum of distances between successive points.

$$
\begin{aligned}
& d=\max \operatorname{dist}\left(P_{1}, P_{i}\right), \\
& L=\sum_{i}^{n-1} \operatorname{dist}\left(P_{1}, P_{i+1}\right) .
\end{aligned}
$$

This method can also be applied to calculate the FD of the mode shapes. Since it exhibits high-noise insusceptibility [37], FD has been applied to crack detection. Crack locations are determined by peaks on the FD curve, which indicates the local irregularity of the fundamental mode shape introduced by the crack. However, inflexions appearing in higher mode shapes would cause false peaks covering up the peaks induced by cracks. Qiao and Cao [38] verified that these false peaks can be inhibited well, using a specific bijective linear mapping from vector space $Z$ to $Z^{*}$ as shown in

$$
\begin{aligned}
& {\left[\begin{array}{l}
x_{i}^{*} \\
y_{i}^{*}
\end{array}\right]=\left[\begin{array}{cc}
1 & 0 \\
\sin \alpha & \cos \alpha
\end{array}\right]\left[\begin{array}{l}
x_{i} \\
y_{i}
\end{array}\right],} \\
& i=1,2, \ldots, n ; \quad-\frac{\pi}{2}<\alpha<\frac{\pi}{2},
\end{aligned}
$$

where $\left(x_{i}, y_{i}\right)$ and $\left(x_{i}^{*}, y_{i}^{*}\right)$ are the vectors in vector spaces $Z$ and $Z^{*}$, respectively. In this paper, $x_{i}$ and $x_{i}^{*}$ denote beam length and $y_{i}$ and $y_{i}^{*}$ certain order mode shape data in vector spaces $Z$ and $Z^{*}$, respectively. $\alpha$ is within interval $(-\pi / 2, \pi / 2)$. And the selection of $\alpha$ is usually done by trial and error.

\section{Numerical Simulations}

4.1. Crack Detection Method. This paper proposes a hybrid method to detect crack locations based on wavelet transform and FD for beam structure. The steps of the proposed method are followed.

4.1.1. Obtain the First Several Mode Shapes of the Cracked Beam. The cracked beam is modeled using the B-spline wavelet on the interval (BSWI) finite element method. The beam crack is modeled according to linear elastic fracture mechanics theory. The first several mode shapes of the cracked beam is acquired from eigen formulation.

4.1.2. Decompose Mode Shape Using 1D Daubechies Wavelet Transform. By Db4 wavelet transform, the wavelet coefficients are calculated for the mode shape. Applying the wavelet coefficients and reconstructing the original mode shapes, approximation signal in scaling space and detailed signals in wavelet spaces are gained.

4.1.3. Calculate FD of Detailed Signals. FD estimation method is used to calculate FD values of detailed signal.

4.1.4. Distinguish Crack Locations. The mode shapes, approximation and detailed signals, and FD are plotted in the geometry space of the beam structure. The crack locations can be identified by the peak points of the signals along the beam length.

4.2. Numerical Examples. Numerical simulation is carried out to verify the effectiveness of the proposed method for a cantilevered steel beam with two cracks. The cantilever beam length $L=0.5 \mathrm{~m}$, cross section $b \times h=0.012 \mathrm{~m} \times 0.019 \mathrm{~m}$, and material parameters are Young's modulus $E=2.06 \times$ $10^{11} \mathrm{~N} / \mathrm{m}^{2}$ and material density $\rho=7860 \mathrm{~kg} / \mathrm{m}^{3}$. The crack parameters are $\alpha_{1}=0.1, \beta_{1}=0.4$, and $\alpha_{2}=0.15, \beta_{2}=0.6$.

The BSWI4 $4_{3}$ Euler beam element is used as approximation bases to model the cracked beam, where 4 and subscript 3 denote the order and the level of the BSWI wavelet. In the simulation, we use $20 \mathrm{BSWI}_{3}$ Euler beam element (184 DOFs). The left of the beam is fixed and its right is free. In this paper, we do not consider damping.

The first three mode shapes of the cracked beam are acquired from eigen formulation. For comparison purpose, mode curvature-based method and the proposed method are adopted to detected crack locations.

The size of sliding window is a crucial parameter, which significantly affects the results of crack identification. And the selection of the size of sliding window is usually done by trial and error. In this paper, the size of sliding window is set as $5 \mathrm{~mm}$. 

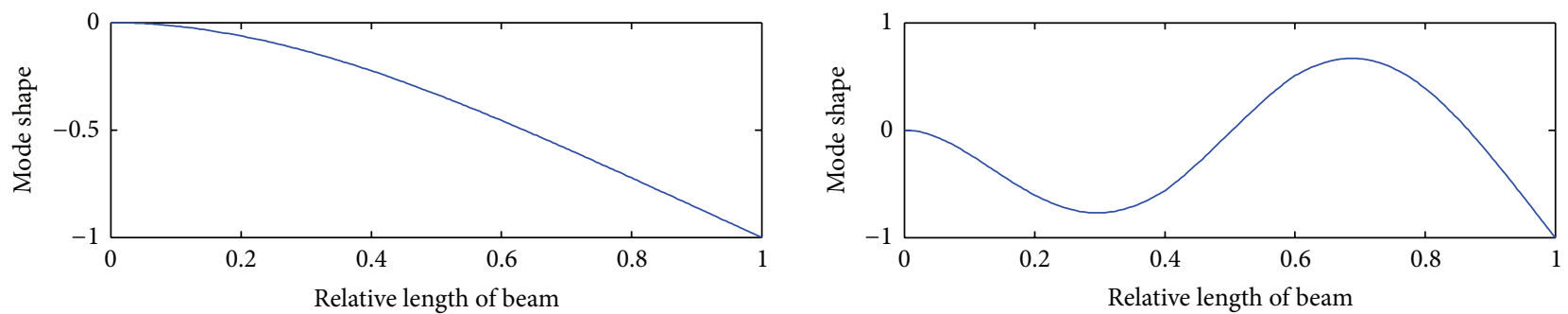

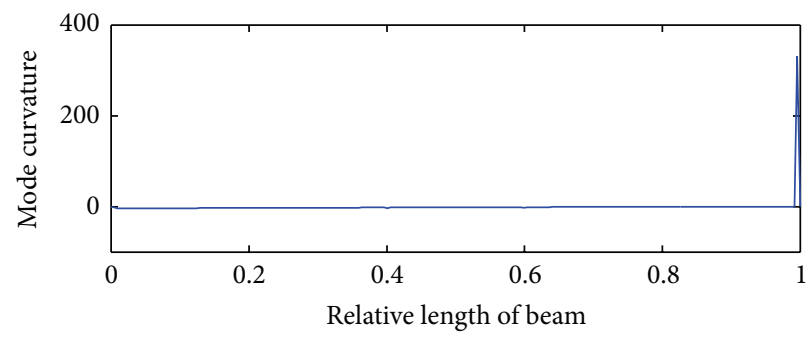

(a)

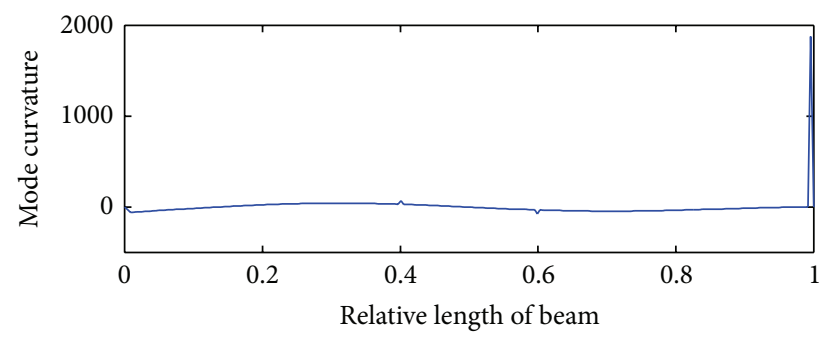

(b)

FIGURE 2: Crack location identification results using mode curvature-based method. (a) The first mode shape. (b) The third mode shape.
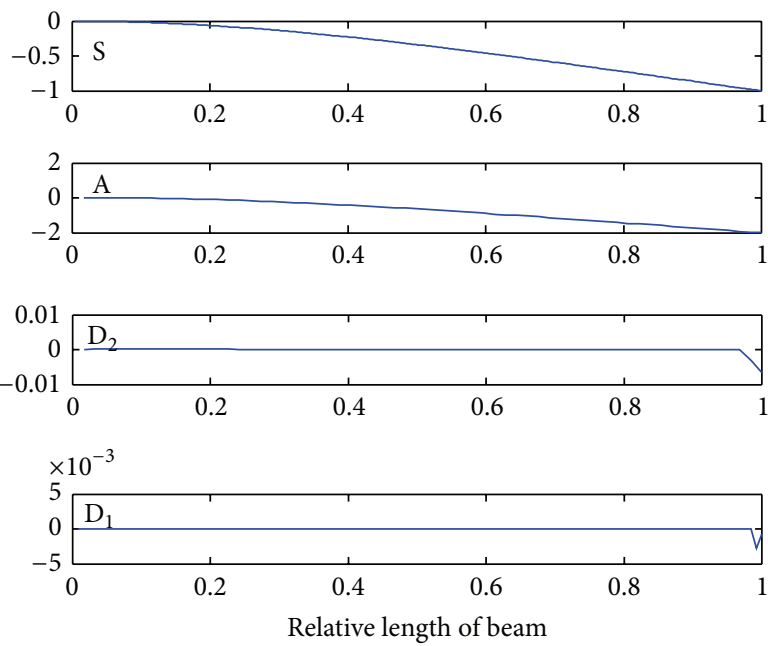

(a)

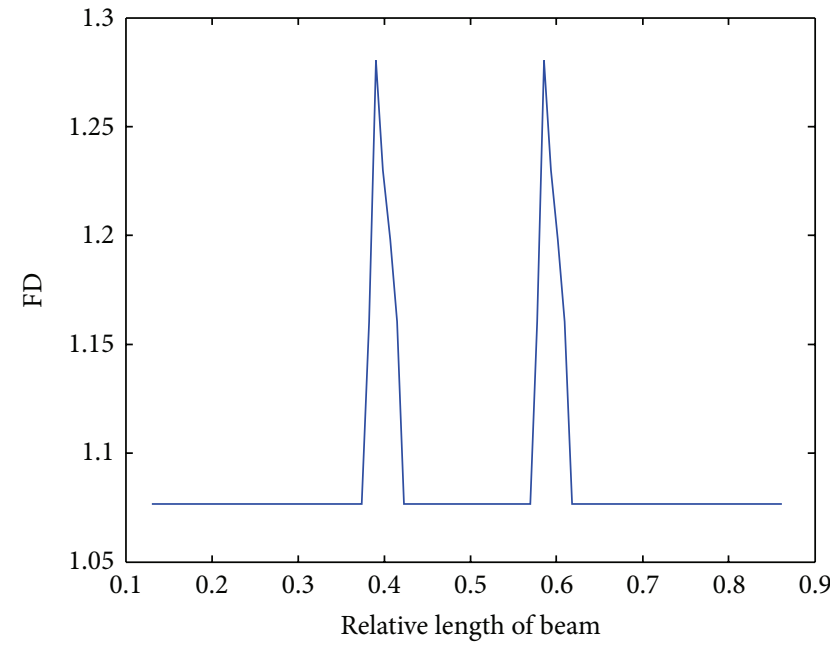

(b)

FIGURE 3: Crack location identification results using the proposed method for the first mode shape. (a) The first mode shape S and its wavelet decomposition at level 2. (b) FD of the detailed signal $\mathrm{D}_{1}$.

Mode curvature-based method is applied to the first and third mode shapes, respectively. The detection results are show in Figure 2. It can be seen that the crack locations are not identified clearly. By comparison, it is seen that the proposed method is more effective to detect crack locations than mode curvature-based method.

The proposed method is used to detect crack locations. The detection results of crack locations are showed in Figures 3 and 4 using the first mode shape and the third mode shape. The mode shape $S$, the approximation signal $A$, and the detailed signals $D_{2}$ and $D_{1}$ are showed in Figures 3(a) and 4(a), respectively. According to Figure 3(b), the relative locations of two cracks are clearly identified by the peak points at $\beta_{1}=0.4$ and $\beta_{2}=0.6$, respectively. However, from Figure $3(\mathrm{a})$, crack locations are not detected from approximation signals and detailed signals.

The proposed method is directly applied to third mode shape, crack locations are shadowed by two false peaks induced by two inflexions of the third mode shape, as showed in Figure 4(b). As mentioned above, a linear mapping as shown in (7) was applied to solve the inflexion problem. The parameter $\varphi$ is selected as $-89^{\circ}$. After a linear mapping, the detection result is displayed in Figure 4(c), and the crack locations are accurately identified at $\beta_{1}=0.4$ and $\beta_{2}=0.6$. 

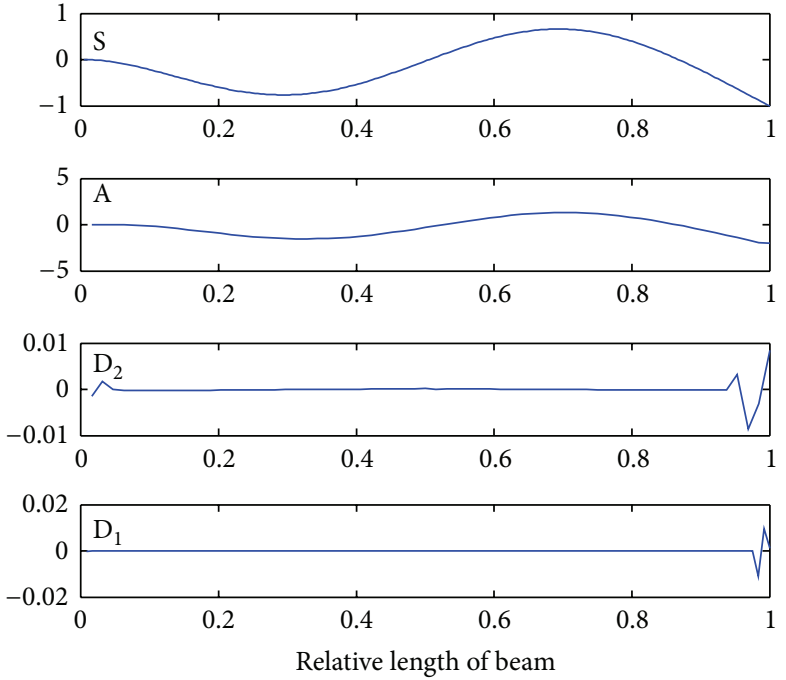

(a)

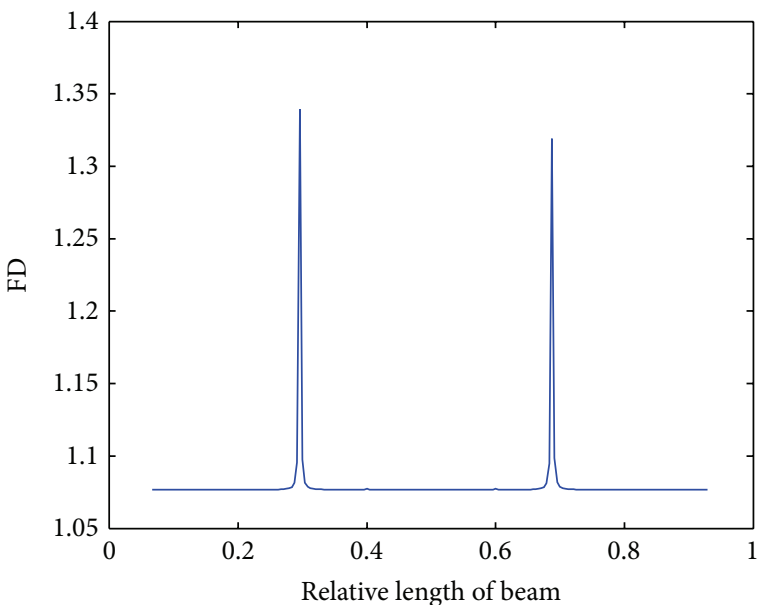

(b)

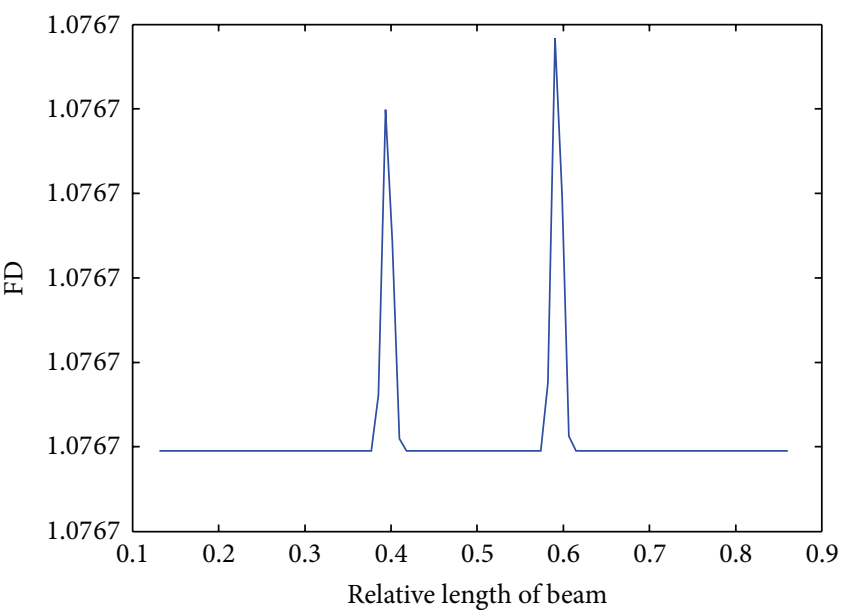

(c)

FIGURE 4: Crack location identification results using the proposed method for the third mode shape. (a) The third mode shape $S$ and its wavelet decomposition at level 2. (b) FD of the detailed signal $\mathrm{D}_{1}$ without linear mapping. (c) FD of the detailed signal $\mathrm{D}_{1}$ by linear mapping.

However, from Figure 4(a), singular locations induced by small beam crack cannot be detected from approximation signal and detailed signals.

By comparison, it is concluded that the proposed method can detect crack locations more accurately than mode curvature-based method and wavelet-based method.

4.3. Noise Immunity. Noise immunity is a vital characteristic to crack detection method. To study noise immunity of the proposed method, Gaussian random white noise with SNR $=80$ and $100 \mathrm{~dB}$ is added to mode shape to simulate noise environment, where SNR is abbreviations of signal-to-noise ratio. A linear mapping as shown in (7) was applied to solve the inflexion problem. The parameter $\varphi$ is selected as a constant near $-89^{\circ}$. Since wavelet transform has multiresolution analysis characteristics. And wavelet transform db 4 at level 2 is used to decompose mode shape to obtain highfrequency detail signal $D_{1}$ and low-frequency approximation signal $\mathrm{A}$ and detail signal $\mathrm{D}_{2}$. In 80 and $100 \mathrm{~dB}$ noise level, the detection results are showed in Figures 5-8. From Figures $5-8$, crack locations are accurately detected at $\beta_{1}=0.4$ and $\beta_{2}=0.6$, respectively. It can be concluded that the proposed method performed well below $80 \mathrm{~dB}$ noise level.

\section{Experimental Validations}

In this section, an experiment is conducted to validate the proposed method on steel cantilever beam with two cracks. The experimental setup is shown in Figure 9. The test system consists of a Polytec Vibrometer PSV-400 and its control system, a shaker, a power amplifier, and a cantilever beam with two cracks.

The geometry of the cantilever beam is shown in Figure 1. The geometry of the cantilever beam is length $L=0.5 \mathrm{~m}$, cross section $b \times h=0.02 \mathrm{~m} \times 0.02 \mathrm{~m}$. And material parameters are Young's modulus $E=2.06 \times 10^{11} \mathrm{~N} / \mathrm{m}^{2}$ and material density 

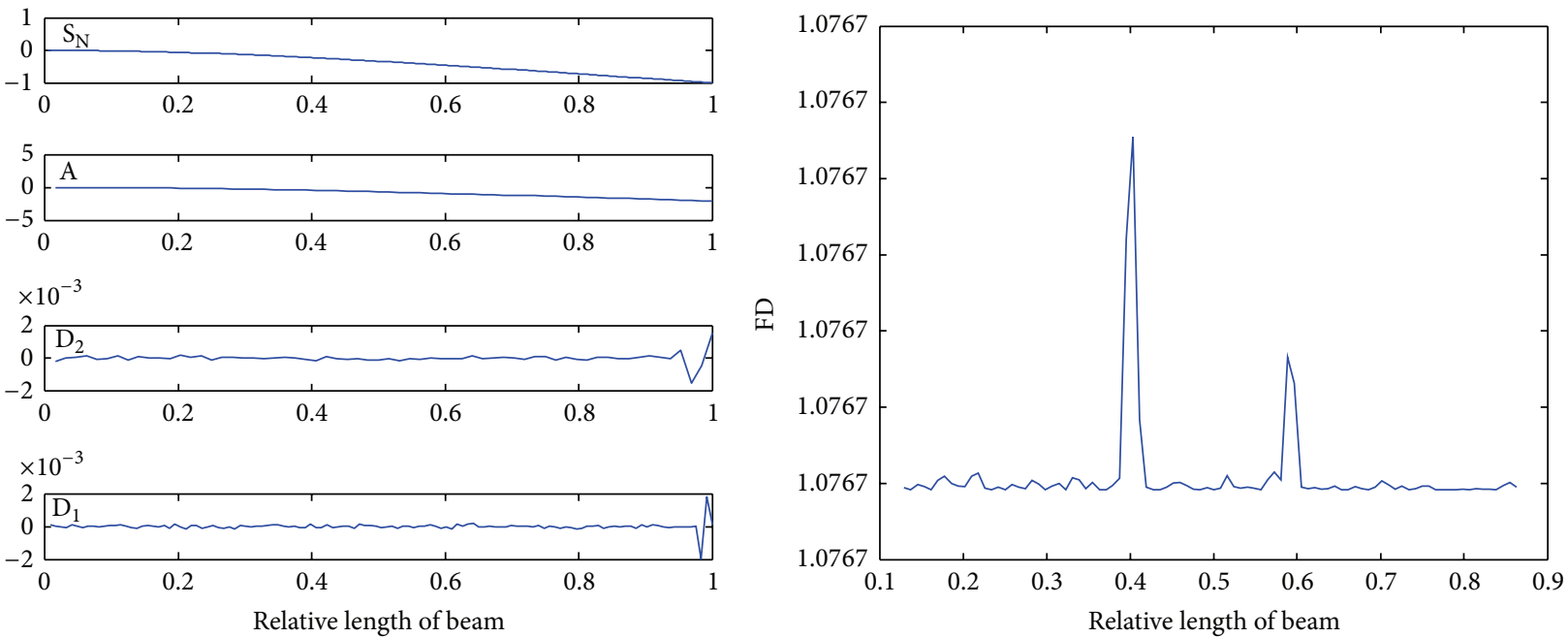

(a)

(b)

FIGURE 5: Crack location identification results in $80 \mathrm{~dB}$ noise level for the first mode shape. (a) Noise contaminated the first mode shape $\mathrm{S}_{\mathrm{N}}$ and its wavelet decomposition at level 2. (b) FD of the detailed signal $\mathrm{D}_{1}$.
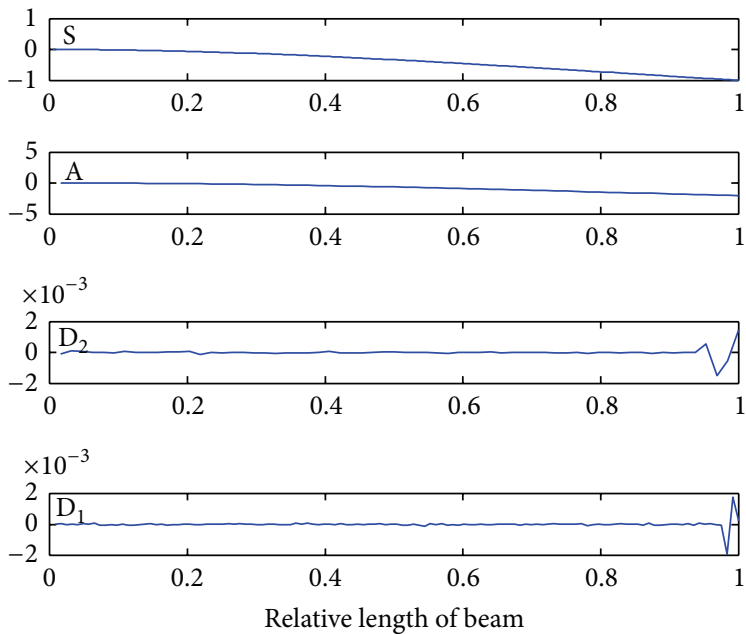

(a)

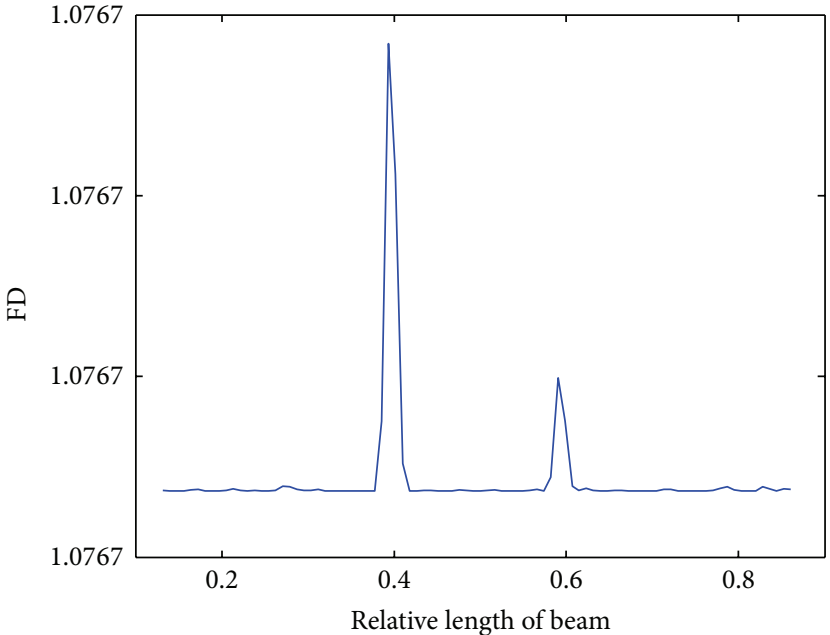

(b)

Figure 6: Crack location identification results in $100 \mathrm{~dB}$ noise level for the first mode shape. (a) Noise contaminated the first mode shape $\mathrm{S}_{\mathrm{N}}$ and its wavelet decomposition at level 2. (b) FD of the detailed signal $\mathrm{D}_{1}$.

$\rho=7860 \mathrm{~kg} / \mathrm{m}^{3}$. The artificial cracks are prepared on the numerical milling machine. The parameters of two cracks are $\alpha_{1}=0.1, \beta_{1}=0.4$ and $\alpha_{2}=0.15, \beta_{2}=0.6$.

The beam is fixed in its left by a holder and connected with electrodynamic mode shaker JMJ-5 near the right, which excites the investigated specimen by the random noise signal. The displacements are acquired using scanning laser Doppler vibrometer (LDV) Polytec PSV-400 connected with a vibrometer controller OFV-5000. 41 equidistant measuring points are arranged along the cantilever beam. The frequency bandwidth is defined within the range of $0-2.5 \mathrm{kHz}$, the resolution is $1 \mathrm{~Hz}$, the sampling frequency is $6.4 \mathrm{kHz}$, and the sampling time is $40 \mathrm{~ms}$.
The first several mode shapes can be acquired by the vibrometer-dedicated software. The first mode shape and the third mode shape are processed based on the proposed method. The experiment results are showed in the Figures 10 and 11, respectively. In Figures 10(a) and 11(a), solid line denotes the FE mode shapes, and black dot denotes the measured mode shapes. By comparison, the first FE mode shape and the first measured mode shape are more fit than the third FE mode shape and the third measured mode shape. The reason resulting in this phenomenon is that the third mode shape is more sensitive to environmental noise and measuring error than the first mode shape. From Figure 10, the crack locations are at $\beta_{1}{ }^{*}=0.41$ and $\beta_{2}{ }^{*}=0.58$ using 

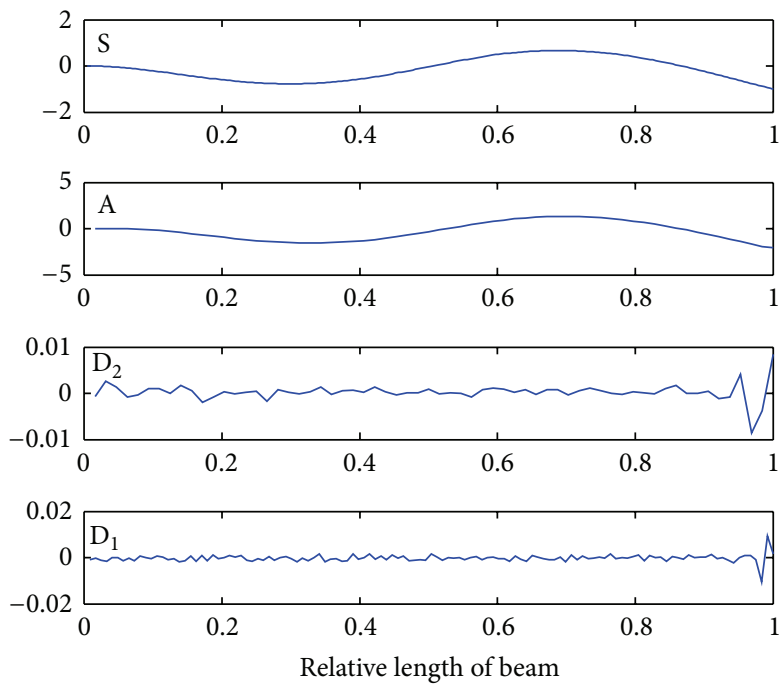

(a)

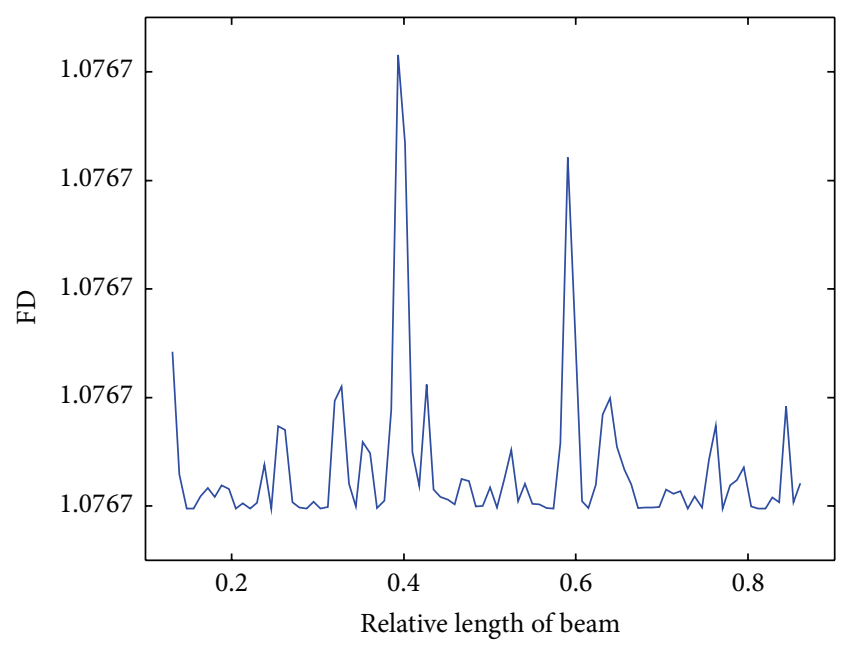

(b)

FIGURE 7: Crack location identification results in $80 \mathrm{~dB}$ noise level for the third mode shape. (a) Noise contaminated the first mode shape $\mathrm{S}_{\mathrm{N}}$ and its wavelet decomposition at level 2. (b) FD of the detailed signal $\mathrm{D}_{1}$.
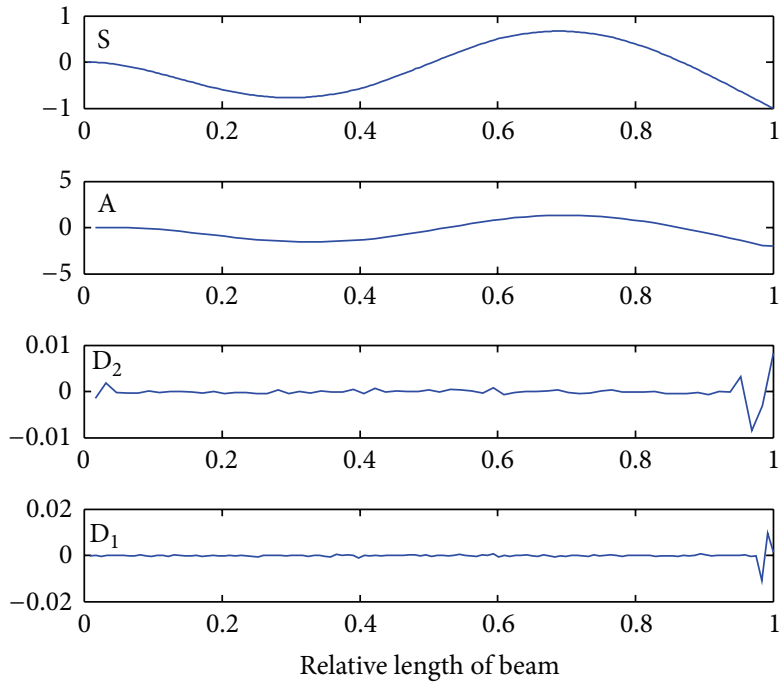

(a)

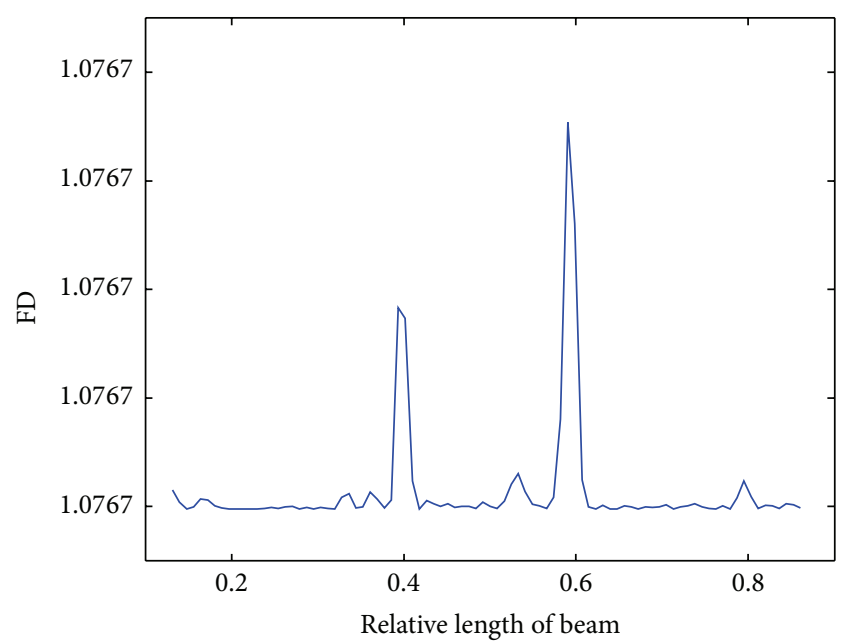

(b)

FIGURE 8: Crack location identification results in $100 \mathrm{~dB}$ noise level for the third mode shape. (a) Noise contaminated the first mode shape $\mathrm{S}_{\mathrm{N}}$ and its wavelet decomposition at level 2. (b) FD of the detailed signal $\mathrm{D}_{1}$.

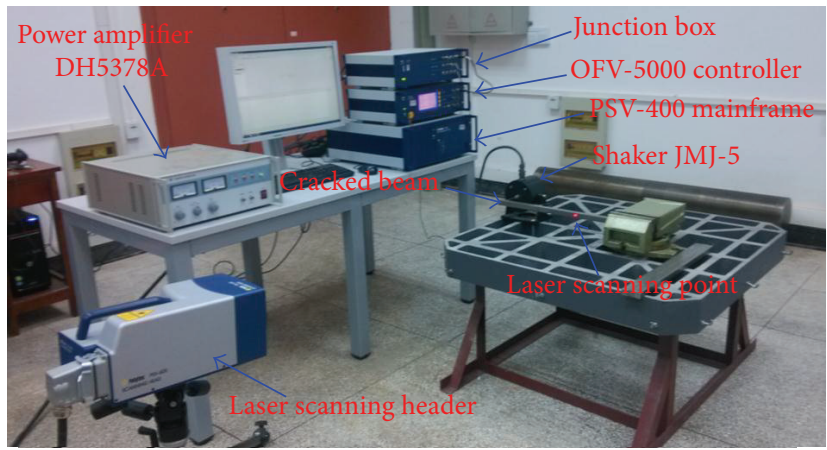

FIGURE 9: Experimental setup. 

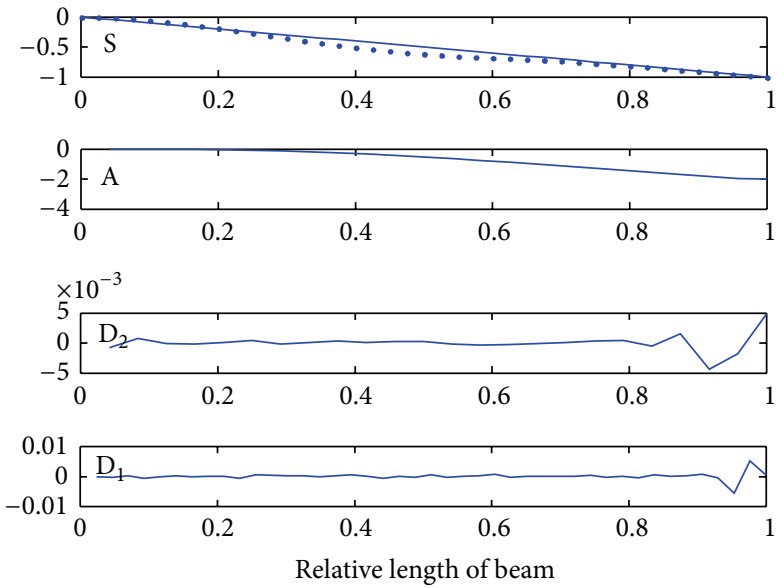

(a)

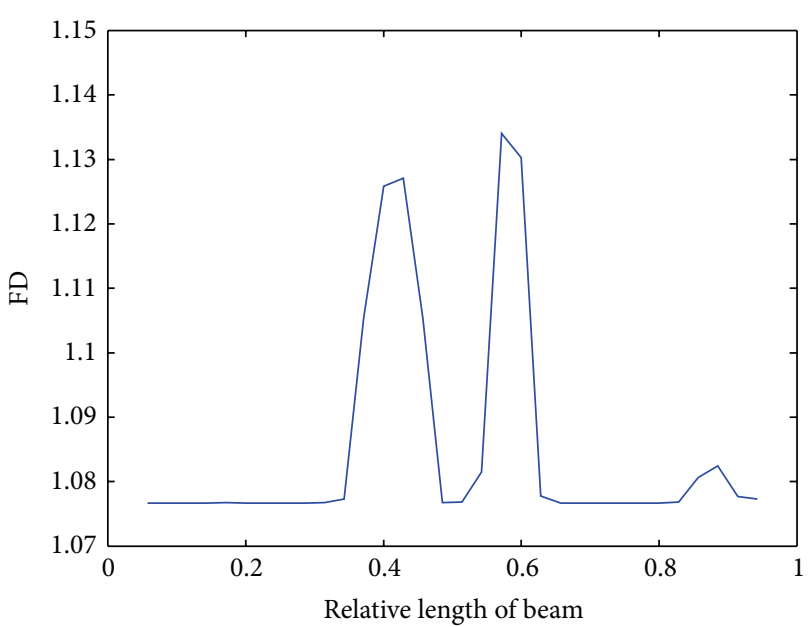

(b)

FIGURE 10: Crack location identification results in experimental circumstances. (a) The first measured mode shape S and its wavelet decomposition at level 2. (b) FD of the detailed signal $\mathrm{D}_{1}$.
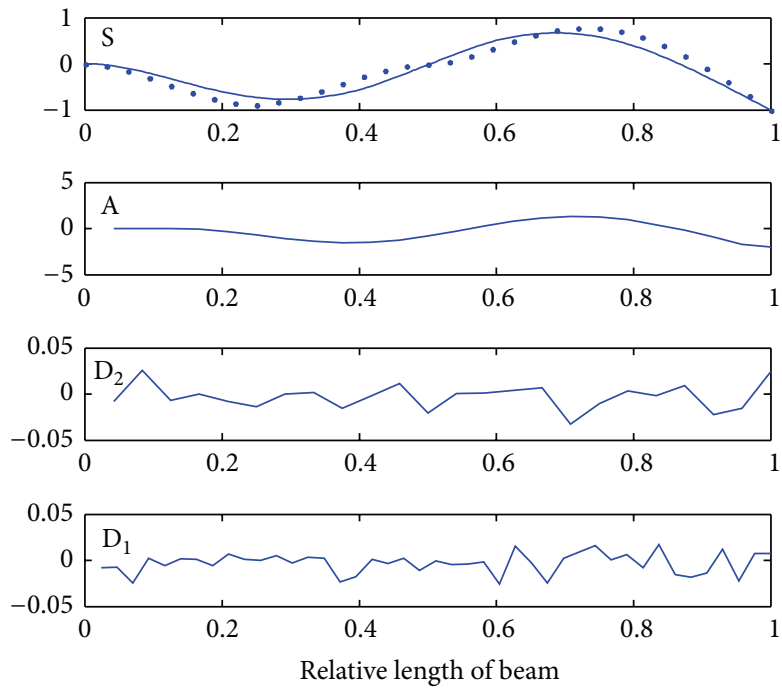

(a)

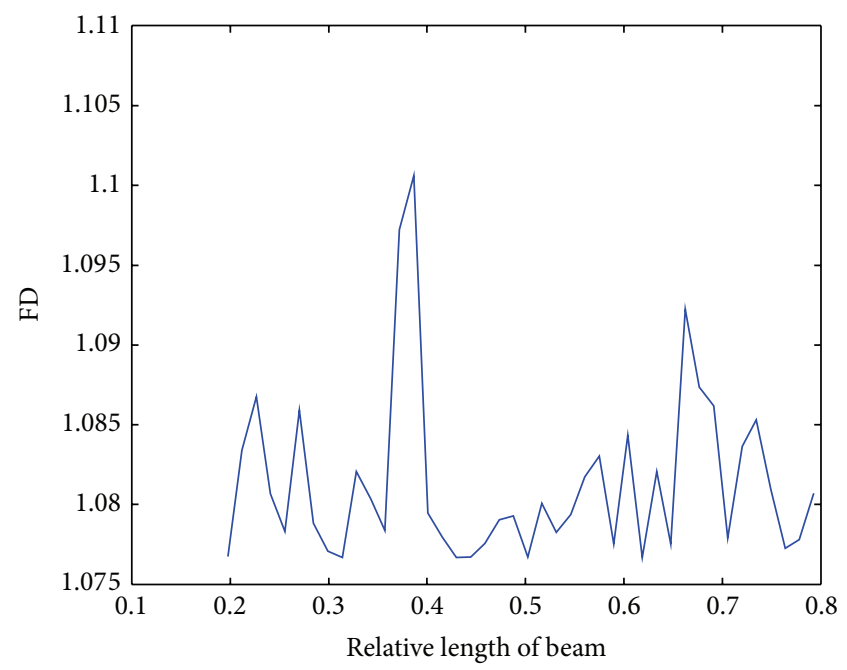

(b)

FIGURE 11: Crack location identification results in experimental circumstances. (a) The third measured mode shape S and its wavelet decomposition at level 2. (b) FD of the detailed signal $\mathrm{D}_{1}$.

the first mode shape, whose relative error is $\varepsilon_{1}=\mid \beta_{1}{ }^{*}-$ $\beta_{1} \mid / \beta_{1}=2.5 \%$ and $\varepsilon_{2}=\left|\beta_{2}{ }^{*}-\beta_{2}\right| / \beta_{2}=3.3 \%$, respectively. From Figure 11, the crack locations are at $\beta_{1}{ }^{*}=0.38$ and $\beta_{2}{ }^{*}=0.66$ using the third mode shape, whose relative error is $\varepsilon_{1}=\left|\beta_{1}{ }^{*}-\beta_{1}\right| / \beta_{1}=5 \%$ and $\varepsilon_{2}=\left|\beta_{2}{ }^{*}-\beta_{2}\right| / \beta_{2}=$ $10 \%$, respectively. According to the experimental results, the relative errors of crack location estimations are within $10 \%$. Hence, the proposed method is effective and can be used for real applications with reasonable accuracy. However, by comparison, it can conclude that the detection accuracy is higher using the first mode shape than using the third mode shape, since high mode shape is more sensitive to environmental noise and measuring error than low mode shape.

\section{Conclusions}

In the present study, a hybrid method is presented to detect crack locations using wavelet transform and fractal dimension (FD) for beam structures. Wavelet transform is applied to decompose the mode shapes of beam structures. To improve the sensitivity of location detection, FD estimation method is employed to analyze detailed signal of the mode shape. For comparison purpose, curvature mode shape based method is also used to identify the crack locations. Numerical simulations and experimental investigations are carried out to test effectiveness of the proposed method for a cantilever beam with two cracks. The results indicate that, using wavelet decomposition or curvature mode alone, the locations of 
small cracks are not detected for beam structures but are identified accurately according to FD singularity of detailed signal. At the same time, the proposed method performs well below $80 \mathrm{~dB}$ level noise and can been extended more complex structures. It is worth to point out that one mode shape should be identified using experimental modal analysis, which is only suitable for offline crack detection. However, for the structures under working condition, many mode shapes will be measured simultaneously to generate the operating deflection shape (ODS). Therefore, the further work is to extend the present approach for online detection of cracks in structures.

\section{Conflict of Interests}

The authors declare that there is no conflict of interests regarding the publication of this paper.

\section{Acknowledgments}

This work was supported by the National Basic Research Program of China ("973" Program) no. 2011CB706805, the National Natural Science Foundation of China nos. 51475356, and Zhejiang Province Public Welfare Project no. $2014 \mathrm{C} 31103$.

\section{References}

[1] Y. Wang and M. Liang, "An adaptive SK technique and its application for fault detection of rolling element bearings," Mechanical Systems and Signal Processing, vol. 25, no. 5, pp. 1750-1764, 2011.

[2] S. W. Doebling, C. R. Farrar, M. B. Prime, and D. W. Shevitz, "A review of damage identification methods that examine changes in dynamic properties," Shock and Vibration Digest, vol. 30, no. 2, pp. 91-105, 1998.

[3] Q. Huang, P. Gardoni, and S. Hurlebaus, "A probabilistic damage detection approach using vibration-based nondestructive testing," Structural Safety, vol. 38, pp. 11-21, 2012.

[4] S. S. Kourehli, G. G. Amiri, M. Ghafory-Ashtiany, and A. Bagheri, "Structural damage detection based on incomplete modal data using pattern search algorithm," JVC/Journal of Vibration and Control, vol. 19, no. 6, pp. 821-833, 2013.

[5] W. Fan and P. Qiao, "Vibration-based damage identification methods: a review and comparative study," Structural Health Monitoring, vol. 10, no. 1, pp. 83-111, 2011.

[6] Y. Wang and M. Liang, "Identification of multiple transient faults based on the adaptive spectral kurtosis method," Journal of Sound and Vibration, vol. 331, no. 2, pp. 470-486, 2012.

[7] H. Saffari, R. Tabatabaei, and S. H. Mansouri, "Vibration analysis of circular arch element using curvature," Shock and Vibration, vol. 15, no. 5, pp. 481-492, 2008.

[8] D. P. Patil and S. K. Maiti, "Detection of multiple cracks using frequency measurements," Engineering Fracture Mechanics, vol. 70, no. 12, pp. 1553-1572, 2003.

[9] Y. Wang, Z. He, and Y. Zi, "A comparative study on the local mean decomposition and empirical mode decomposition and their applications to rotating machinery health diagnosis," Journal of Vibration and Acoustics, Transactions of the ASME, vol. 132, no. 2, Article ID 021010, 2010.
[10] Z. Yang, X. Chen, J. Yu, R. Liu, Z. Liu, and Z. He, "A damage identification approach for plate structures based on frequency measurements," Nondestructive Testing and Evaluation, vol. 28, no. 4, pp. 321-341, 2013.

[11] H. Y. Guo and Z. L. Li, "A two-stage method for damage detection using frequency responses and statistical theory," Journal of Vibration and Control, vol. 18, no. 2, pp. 191-200, 2012.

[12] J. W. Xiang and M. Liang, "Wavelet-based detection of beam cracks using modal shape and frequency measurements," Computer-Aided Civil and Infrastructure Engineering, vol. 27, no. 6, pp. 439-454, 2012.

[13] M. R. Naniwadekar, S. S. Naik, and S. K. Maiti, "On prediction of crack in different orientations in pipe using frequency based approach," Mechanical Systems and Signal Processing, vol. 22, no. 3, pp. 693-708, 2008.

[14] M. Lepidi, V. Gattulli, and F. Vestroni, "Damage identification in elastic suspended cables through frequency measurement," Journal of Vibration and Control, vol. 15, no. 6, pp. 867-896, 2009.

[15] F. B. Sayyad and B. Kumar, "Identification of crack location and crack size in a simply supported beam by measurement of natural frequencies," Journal of Vibration and Control, vol. 18, no. 2, pp. 183-190, 2012.

[16] A. Morassi and M. Rollo, "Identification of two cracks in a simply supported beam from minimal frequency measurements," Journal of Vibration and Control, vol. 7, no. 5, pp. 729-739, 2001.

[17] E. Douka, S. Loutridis, and A. Trochidis, "Crack identification in plates using wavelet analysis," Journal of Sound and Vibration, vol. 270, no. 1-2, pp. 279-295, 2004.

[18] J. W. Xiang and M. Liang, "A two-step approach to multidamage detection for plate structures," Engineering Fracture Mechanics, vol. 91, pp. 73-86, 2012.

[19] A. B. Stanbridge, A. Z. Khan, and D. J. Ewins, "Modal testing using impact excitation and a scanning LDV," Shock and Vibration, vol. 7, no. 2, pp. 91-100, 2000.

[20] W. L. Bayissa, N. Haritos, and S. Thelandersson, "Vibrationbased structural damage identification using wavelet transform," Mechanical Systems and Signal Processing, vol. 22, no. 5, pp. 1194-1215, 2008.

[21] W.-X. Ren and Z.-S. Sun, "Structural damage identification by using wavelet entropy," Engineering Structures, vol. 30, no. 10, pp. 2840-2849, 2008.

[22] S. Loutridis, E. Douka, L. J. Hadjileontiadis, and A. Trochidis, "A two-dimensional wavelet transform for detection of cracks in plates," Engineering Structures, vol. 27, no. 9, pp. 1327-1338, 2005.

[23] F. Li, G. Meng, K. Kageyama, Z. Su, and L. Ye, "Optimal mother Wavelet selection for lamb wave analyses," Journal of Intelligent Material Systems and Structures, vol. 20, no. 10, pp. 1147-1161, 2009.

[24] F. C. Li, H. Murayama, K. Kageyama, and T. Shirai, "Guided wave and damage detection in composite laminates using different fiber optic sensors," Sensors, vol. 9, no. 5, pp. 40054021, 2009.

[25] D. Chendong and G. Qiang, "A lifting undecimated wavelet transform and its applications," Journal of Intelligent Manufacturing, vol. 19, no. 4, pp. 433-441, 2008.

[26] R. Yan and R. X. Gao, "Wavelet domain principal feature analysis for spindle health diagnosis," Structural Health Monitoring, vol. 10, no. 6, pp. 631-642, 2011. 
[27] R. Yan and R. X. Gao, "Harmonic wavelet-based data filtering for enhanced machine defect identification," Journal of Sound and Vibration, vol. 329, no. 15, pp. 3203-3217, 2010.

[28] L. J. Hadjileontiadis, E. Douka, and A. Trochidis, "Fractal dimension analysis for crack identification in beam structures," Mechanical Systems and Signal Processing, vol. 19, no. 3, pp. 659674, 2005.

[29] L. J. Hadjileontiadis and E. Douka, "Crack detection in plates using fractal dimension," Engineering Structures, vol. 29, no. 7, pp. 1612-1625, 2007.

[30] Z. Yang, X. Chen, S. Tian, and Z. He, "Multiple damages detection in beam based approximate waveform capacity dimension," Structural Engineering and Mechanics, vol. 41, no. 5, pp. 663673, 2012.

[31] C. Zhang, B. Li, Z. Yang, W. Xiao, and Z. He, "Crack location identification of rotating rotor systems using operating deflection shape data," Science China Technological Sciences, vol. 56, no. 7, pp. 1723-1732, 2013.

[32] H. Tada, P. C. Paris, and G. R. Irwin, The Stress Analysis of Cracks Handbook, vol. 130, ASME Press, New York, NY, USA, 2000.

[33] J. W. Xiang, X. F. Chen, Z. J. He, and H. B. Dong, "The construction of $1 \mathrm{D}$ wavelet finite elements for structural analysis," Computational Mechanics, vol. 40, no. 2, pp. 325-339, 2007.

[34] J. N. Franklin, Matrx Theory, Prentice Hall, New York, NY, USA, 2000.

[35] B. Mandelbrot, "How long is the coast of Britain? Statistical selfsimilarity and fractional dimension," Science, vol. 156, no. 3775, pp. 636-638, 1967.

[36] M. J. Katz, "Fractals and the analysis of waveforms," Computers in Biology and Medicine, vol. 18, no. 3, pp. 145-156, 1988.

[37] R. Esteller, G. Vachtsevanos, J. Echauz, and B. Litt, "A comparison of fractal dimension algorithms using synthetic and experimental data," in Proceedings of the IEEE International Symosium on Circuits and Systems (ISCAS '99), vol. 3, pp. 199202, IEEE, Orlando, Fla, USA, July 1999.

[38] P. Qiao and M. Cao, "Waveform fractal dimension for mode shape-based damage identification of beam-type structures," International Journal of Solids and Structures, vol. 45, no. 22-23, pp. 5946-5961, 2008. 

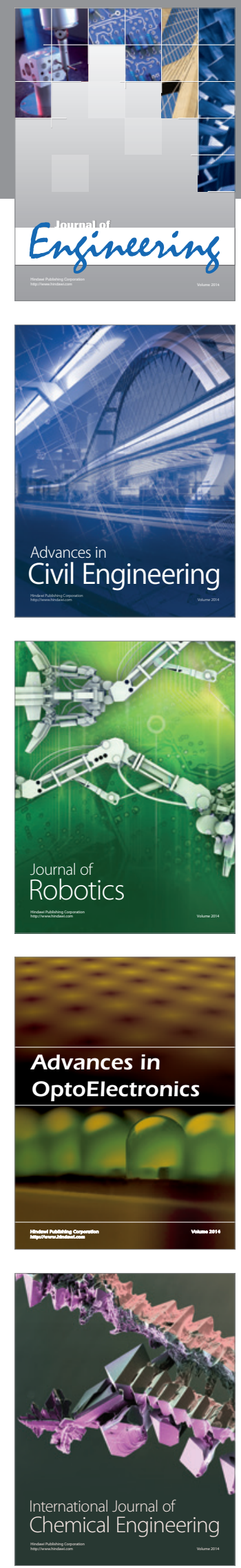

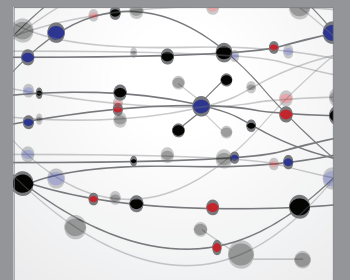

The Scientific World Journal
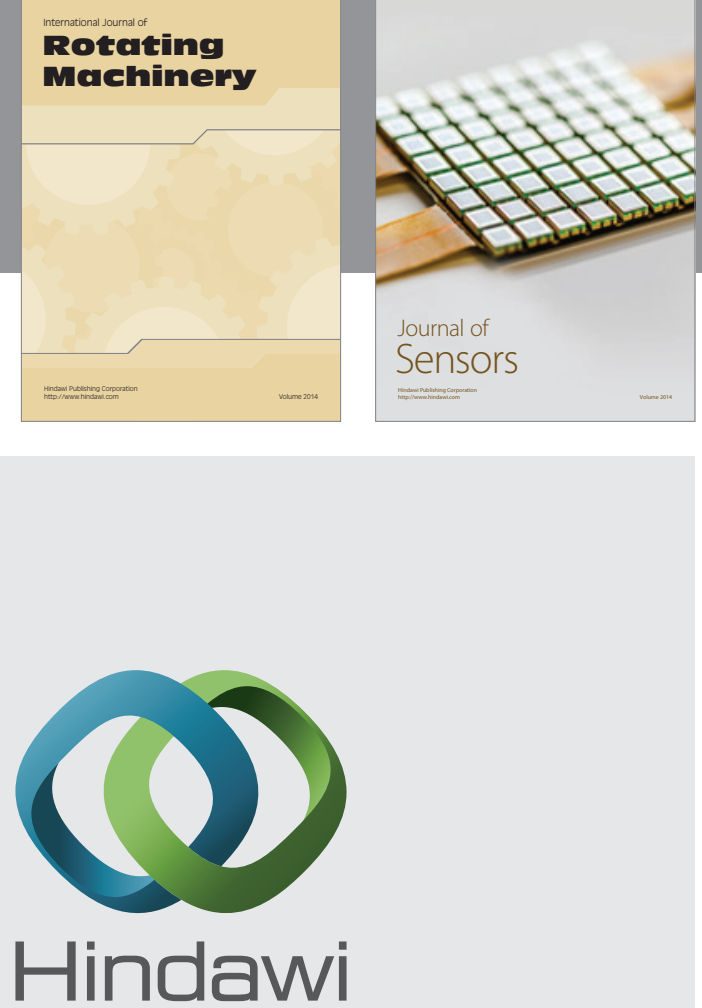

Submit your manuscripts at http://www.hindawi.com
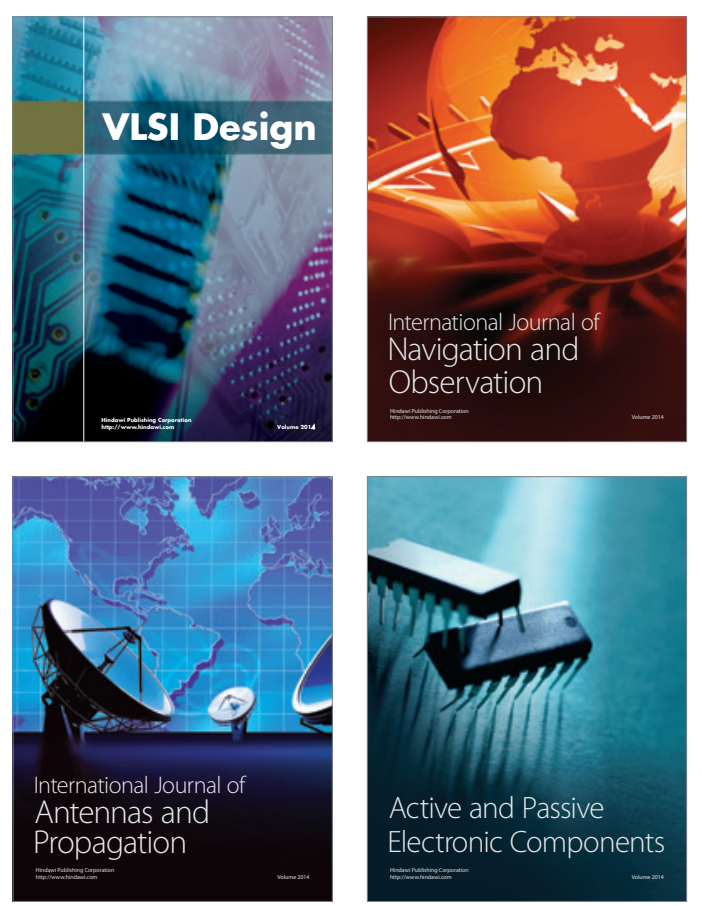
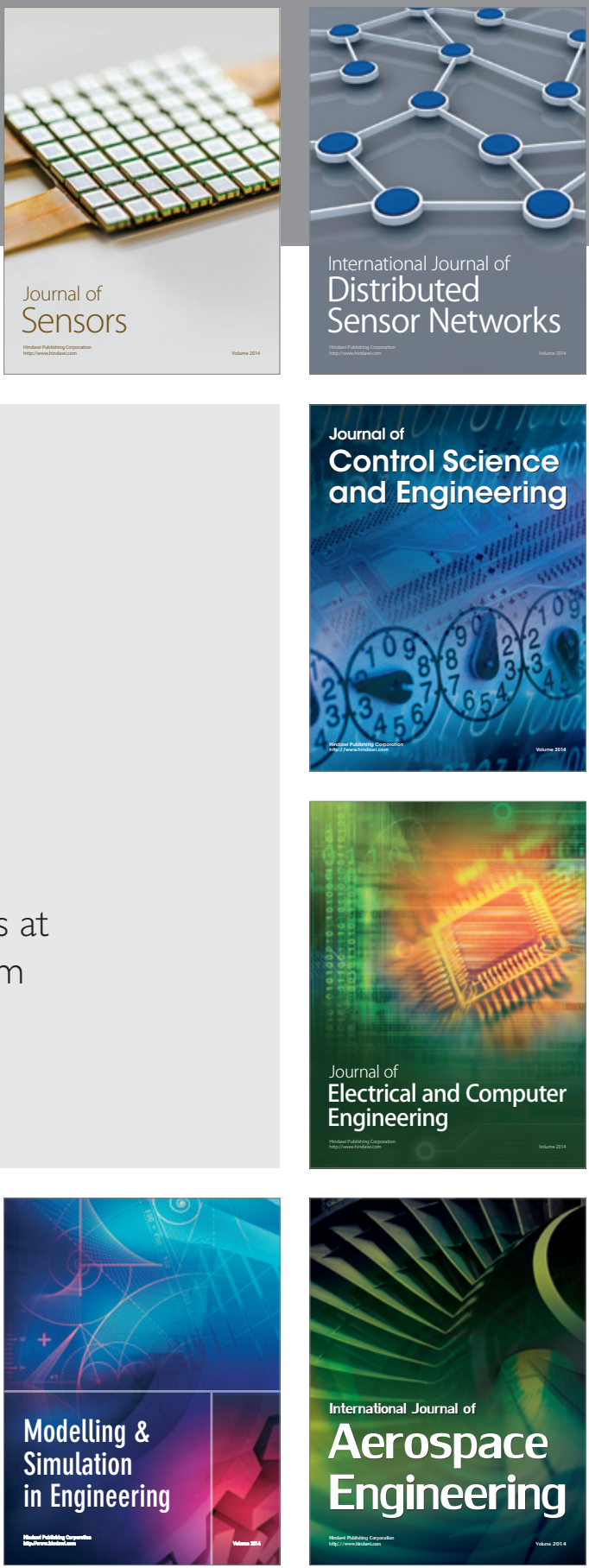

Journal of

Control Science

and Engineering
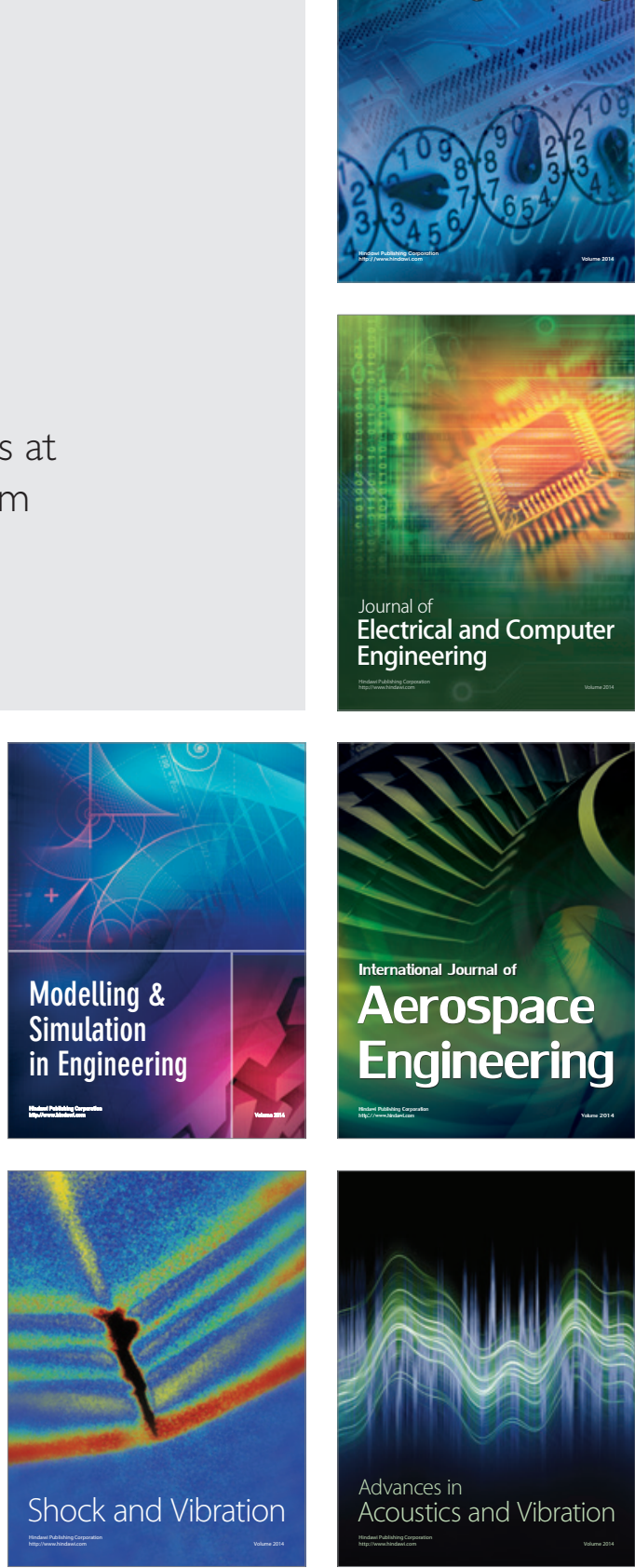